

ISSN: $2395-1958$

IJOS 2019; 5(2): 01-05

(C) 2019 IJOS

www.orthopaper.com

Received: 01-02-2019

Accepted: 04-03-2019

Dr. Lakhwinder Singh

Assistant Professor, Department of Orthopedics, Dr. Ulhas Patil

Medical College Jalgaon,

Maharashtra, India

Dr. Nilesh Kachnerkar

Associate Professor, Department of Orthopaedics, Government

Medical College, Jalgaon,

Maharashtra, India
Correspondence

Dr. Nilesh Kachnerkar

Associate Professor, Department

of Orthopaedics, Government

Medical College, Jalgaon,

Maharashtra, India

\section{Result of cancellous screw fixation in fracture neck of femur in adults}

\author{
Dr. Lakhwinder Singh and Dr. Nilesh Kachnerkar
}

DOI: https://doi.org/10.22271/ortho.2019.v5.i2a.01

\section{Abstract}

Background: Cancellous screw fixation is commonly performed procedures for the management of fracture neck of femur in adults. Cancellous screw fixation is associated with high percentage of excellent and good results and is associated with reduced invasion and short operative time.

Objectives

1. To study the incidence of fracture neck of femur according to various grades of Garden's classification.

2. To study the quality of life after screw fixation using Modified Harris Hip Scoring (MHHS) system.

3. To evaluate the results of screw fixation in various grades of Garden's classification.

Patients and Methods: Adult patients ranging from 20-50 years those suffering from fracture neck of femur were treated by cancellous screw fixation. The study was carried out to evaluate the immediate and early results of cancellous screw fixation for intracapsular fracture neck of femur in young adults.

Results: Study conducted on 50 patients of both sex and age between 20 yrs and 50 yrs within the period of 2 years those suffering from fracture neck of femur and treated by cancellous screw fixation and follow up was done at 6 weeks, 3 months, 6 months and 9 months and Modified Harris Hip Score was noted. Data was tabulated and statistically analysed.

Conclusions: Our study showed $76 \%$ having excellent results, $18 \%$ having good results, $2 \%$ having fair and $4 \%$ poor results. It was found that there is correlation between initial displacement and the final functional scores. In our study, undisplaced fractures (grade I and II) consistently showed excellent and good results whereas displaced fractures (grade III and IV) were associated with fair and poor results also.

Keywords: Fracture neck of femur, cancellous screw, modified harris hip scoring (MHHS)

\section{Introduction}

Hip fractures are devastating injuries that most commonly affects the elderly and have a tremendous impact on both the health care system and society in general. Fracture neck of femur has been recognised since the time of Hippocrates. The first description of hip fractures was given by French surgeon Ambroise Pare in $1564^{[1]}$. However Sir Astley Cooper gave a clear description of fracture neck of femur and other fractures and dislocation about the hip ${ }^{[2]}$. In 1866, Hamilton and Stimson explained the preferential treatment of internal fixation for fracture neck of femur, quoting surgeries performed by John Ray Burton in Philadelphia during $1834^{[3]}$. Whiteman ${ }^{[4,5]}$ and Leadbetter ${ }^{[6]}$ methods of closed reduction were important contribution to conservative management. This however produced a few satisfactory unions, but extremely high morbidity and mortality. In 1908, Davis reported use of ordinary wood screws for the fixation of femoral neck fractures ${ }^{[7]}$. Similar screws were used by Decosta in $1907^{\text {[7] }}$, Delbet in 1909 and Martin and Knight in 1920.

The first effective method of internal fixation was introduced in 1931 by Smith Peterson and associates ${ }^{[8]}$. S-P nail technique was simplified by the introduction of cannulated nail by Johannson in $1932^{[9]}$ and Westcott in $1934^{[10]}$. A cancellous lag screw is currently the most commonly used implant for stabilization of femoral neck fractures. Different types of designs have been used including Von Bahr, Uppasala, Garden Asins and the AO/ASIF screws ${ }^{[11]}$. Protzman and Burkhalter emphasized three basic differences between these femoral neck fractures in adults and those in elderly individual: (a) they are distinctly uncommon; (b) the reported results of treatment are notably poorer than those in elderly patients; and (c) there is 
significant difference in the severity of trauma required to cause this fracture in adults ${ }^{[12]}$.

Eliasson-Eiskjaer and Ostgard [13] and, recently, Kreder [14] demonstrated that classification and net displacement does not alter treatment or outcome in Garden stage I compared with stage II fractures nor in Garden stage III fractures as compared with stage IV fractures [13, 14], because of these findings, they recommended simply distinguishing between undisplaced (Garden I and II) and displaced (Garden III and IV) femoral neck fractures.

The treatment algorithm in elderly consist of either hemiarthroplasty or total hip replacement but in adults the treatment algorithm falls in watershed zone due to two main risks, non-union and avascular necrosis followed by late segmental collapse later on and needing another surgery. Non-union is reported to be rare after undisplaced fracture, but occurs in $20-30 \%$ of displaced fractures. As age advances, the rate of non-union increases sharply. Avascular necrosis is one of the two important complications of femoral neck fractures. Aseptic necrosis is the actual death of bone secondary to ischemia, an early phenomenon after fracture neck of femur and is a microscopic event [15, 16]. Late segmental collapse is the collapse of the subchondral bone and articular cartilage that overlies the infarcted bone. Incidence of late segmental collapse varies from $7 \%$ to $27 \%$. It occurs in 10-20\% of undisplaced fractures and in 15-35\% of displaced fractures. Garden had reported increased frequency in women then in men ${ }^{[17]}$. Other reported complications are related to the internal fixation itself.

A retrospective study of 84 patients was done by st. Pellet et al. (2003) at department of traumatology, university hospital, Lauranne, Switzerland who were operated by cancellous screw for intracapsular fracture neck of femur. The study showed mean age of 63.6 years. The numbers of patients with various Garden grades were as followed: - grade I $-56 \%$, grade II $-19 \%$, grade III $-19 \%$ and grade IV- $6 \%$. The patients were followed for a mean time of 2 years. Follow-up showed complication in 15 patients with AVN being the most common-11 patients $(13.4 \%)$, followed by non-union in patients $(4.8 \%)^{[18]}$.

KBL Lee et al. (2004) in there study evaluated the efficacy and safety of cancellous screw fixation in intracapsular fracture neck of femur in 116 patients. The patients were followed for over 2 years. The study showed the median age of study was 71 years and two-third was females. There were $104(90 \%)$ cases of undisplaced (Grade I and II) fractures and $12(10 \%)$ cases of displaced (Grade III and IV) fractures. At 2 year follow-up, $85 \%$ returned to the pre- morbid level of ambulation and $90 \%$ reported good pain relief. The complication rate was $15 \%$ (11 cases)- 11 showed non-union $(9 \%)$ and in 6 showed AVN $(5 \%)^{[19]}$.

A study of 64 consecutive cases of intracapsular fracture neck of femur was carried out by Lo Irene et al. (2011) in young adults (age $<65$ years) treated with reduction and screw fixation between 2000-2007. The study showed the mean age was 53.5 years (32-65 years). The mean follow-up period was 36.8 months (6-100 months). The male female ratio was almost equal (29 versus 35). The study showed AVN in 9 cases $(14.1 \%)$. There were 2 cases $(3.1 \%)$ of non-union ${ }^{[21]}$.

So this study assesses the functional results of cancellous screw fixation using Modified Harris Hip Scoring (MHHS) and results in various grades of Garden's classification.

\section{Aims and objectives}

1. To study the incidence of fracture neck of femur according to various grades of Garden's classification.

2. To study the quality of life after screw fixation using Modified Harris Hip Scoring (MHHS) system.

3. To evaluate the results of screw fixation in various grades of Garden's classification.

\section{Material and methods}

A study was conducted in 50 consecutive cases of intracapsular fracture neck of femur in patient between the age of 20-50 years which are admitted in the department of orthopaedics treated by cancellous screw fixation irrespective of sex and socio-economic status. The study was carried out to evaluate the immediate and early results of cancellous screw fixation for intracapsular fracture neck of femur in young adults.

\section{Inclusion criteria included}

1. Age between 20-50 years

2. Traumatic femoral neck fracture presenting within 1 week of injury.

3. Patients who are medically fit for surgery.

\section{ASA 1 and 2}

1. Patients with pathological femoral neck fracture.

2. Ipsilateral or contralateral major limb injury affecting treatment and rehabilitation.

Procedure: Patients were admitted to the ward. In all patients buck's traction with 5 pound weight was applied to the fracture limb and patients were given oral or parenteral NSAIDs to relieve the pain. Antero-posterior radiograph of the pelvis with both hips were taken for all the patients, keeping the fractured limb in $15^{\circ}$ internal rotation to bring the neck parallel to X-ray film. Patients are classified using Garden's classification.

All surgeries were performed on an elective basis using standard aseptic precautions. Surgeries were performed under spinal or general anaesthesia on a fracture table where the pelvis was rested on the perineal support and the affected limb was placed in abduction to facilitate $\mathrm{C}$-arm examination (fluoroscopic image intensifier).

Reduction was done by closed method using either Whitman or Leadbetter method whichever succeeds in accurate reduction. The accuracy of reduction was evaluated clinically by the "heel-palm" test and radiographically by Garden's alignment index. In heel-palm test, the heel is placed in the palm of an outstretched hand. If the reduction is complete, the leg will not rotate spontaneously externally. Reduction was confirmed with AP and lateral view under $\mathrm{C}$-arm exposure on monitor screen.

The affected limb was scrubbed from abdomen to ankle joint, then painted with betadine solution and drape applied exposing ASIS and proximal end of lateral part of thigh for the intended procedure.

Surgical technique ${ }^{[21]}$ : as per standard technique a lateral longitudinal incision in line with the greater trochanter measuring about 4-6 cm starting at the base of the greater trochanter and extending distally was made. The superficial fascia, tensor fascia lata and vastus lateralis were then split in the line of skin incision. The origin of vastus lateralis were elevated subperiostelly at the base of greater trochanter.

Using a power drill, a positioning guide wire was placed. Confirming the position of wire with $\mathrm{C}$-arm, wire was placed accurately in the proximal femur, calcar and head parallel to 
the anteversion wire in antero-posterior and lateral views. This wire stabilises the head and prevents displacement or rotation of head during insertion of other guide wires. Second guide wire was placed in the postero-superior part. Third guide wire was placed in the antero-superior part.

The screws were placed as far as possible close to the cortical bone. The anteversion guide was removed. The position of guide wires was confirmed in C-arm image intensifier in both $\mathrm{AP}$ and lateral views. All the guide wires were threaded into the subchondral bone upto $5-10 \mathrm{~mm}$ of the femoral head margin.

Using the direct measuring device, the insertion depth of three guide wires was determined. The drilling depth was calculated by substracting $10 \mathrm{~mm}$ from this reading. This is to prevent penetration of the joint. The screw length was calculated by adding $5 \mathrm{~mm}$ to the drilling depth as the screw head remains outside the near cortex.

$4.0 \mathrm{~mm}$ cannulated drill bit with drill sleeve was inserted over the guide wire. Drilling was done to the depth determined as above and confirmed with image intensifier. Care was taken not to change the direction of drill bit but rather to let it follow the guide wire. Cannulated tap was passed over the guide wire to tap the near cortex.

A screw was selected so that the threaded portion crosses the fracture line and engages the proximal fragment. The screws were used with washers in some cases to prevent screw head penetration into the bone. Using the cannulated hexagonal screw driver, the cannulated screws were inserted over the guide wire till $5-10 \mathrm{~mm}$ of screw was outside the lateral cortex. All the screws were tightened simultaneously for final purchase into the subchondral femoral head. The position of screw was confirmed in C-arm image intensifier in both AP and lateral views.

Post-operative management: In case of spinal anaesthesia, foot end elevation was given depending on the patients postoperative blood pressure. Every 6hrly blood pressure, pulse rate, temperature, respiratory rate monitored for the first 24 hrs. Intramuscular analgesics were given as per patient's compliant, intravenous antibiotics were continued for 3 days [22].

Patients were made to sit up on the first day after surgery. Quadriceps and knee bending started on $2^{\text {nd }}$ day. Check radiograph was taken after 48 hours to note the adequacy of reduction. First dressing was done on $5^{\text {th }}$ post-operative day to observe for any discharge, presence of infection, gaping and condition of stitch line. Suture removal was done on $11^{\text {th }}$ postoperative day. The patients were mobilised using a walker as soon as possible but weight bearing on affected limb was not allowed before discharge from hospital.

Patients who had infection and bed sores were treated accordingly before discharging them from the hospital. After suture removal, the patients were discharged with instructions not to bear weight on the affected limb till further instruction. Sitting cross-legged, squatting and straight leg raising were not allowed. Patients were told to continue static quadriceps and knee bending exercises and to do the toe and ankle movement.

At the time of discharge the patients were asked to come for follow up after 6 weeks and for further follow up at 3 months, 6 months and 9 months. The patients who turned for follow up or whose details could be collected were finally taken up for assessment for functional results. At each follow up patients were evaluated clinically by Modified Harris Hip Scoring system and clinical examination was done regarding the pain, tenderness, active range of movements of hip and limb length discrepancy and all the details were recorded in the follow up chart. The radiograph of operated hip was taken at each follow up and looked for progressive signs of union, and accordingly patients were allowed partial to full weight bearing walking.

At 9 months patients were evaluated clinically and final MHHS was noted and radiologically union and complications like non-union, AVN (evident by area of increased density in subchondral area), any loosening of threads or shaft of screws, breakage of screws, migration into acetabulum, neck length absorption as evident by prominence or backing out of screw heads, loss of reduction with screw in place were noted.

\section{Results}

Study conducted on 50 patients of both sex and age between 20 yrs and 50 yrs, treated by internal fixation by cancellous screw were subjected for this study and patients were followed at the interval of 6 weeks, 3 months, 6 months and 9 month at follow up. Data was tabulated and statistically analysed.

Table 1: Showing age distribution

\begin{tabular}{|c|c|}
\hline & Age \\
\hline Mean & 36.6 \\
\hline S.D. & 9.08 \\
\hline
\end{tabular}

Table 2: Showing sex distribution

\begin{tabular}{|c|c|c|c|c|}
\hline \multirow{3}{*}{ Sex } & \multicolumn{2}{|c|}{ Males } & \multicolumn{2}{c|}{ Females } \\
\cline { 2 - 5 } & Frequency & $\%$ & Frequency & $\%$ \\
\cline { 2 - 5 } & 39 & 78.00 & 11 & 22.00 \\
\hline
\end{tabular}

Table 3: Showing side involvement

\begin{tabular}{|c|c|c|c|c|}
\hline \multirow{3}{*}{ Side } & \multicolumn{2}{|c|}{ Right } & \multicolumn{2}{c|}{ Left } \\
\cline { 2 - 5 } & Frequency & $\%$ & Frequency & $\%$ \\
\cline { 2 - 5 } & 23 & 46.00 & 27 & 54.00 \\
\hline
\end{tabular}

Table 4: Showing fracture type according to Garden's classification

\begin{tabular}{|c|c|c|}
\hline Garden's classification & No. of cases & Percentage (\%) \\
\hline I & 7 & 14 \\
\hline II & 10 & 20 \\
\hline III & 8 & 16 \\
\hline IV & 25 & 50 \\
\hline
\end{tabular}

Table 5: Showing results according to Modified Harris Hip Scoring system

\begin{tabular}{|c|c|c|}
\hline Results & No. of cases & Percentage (\%) \\
\hline Excellent & 38 & 76 \\
\hline Good & 9 & 18 \\
\hline Fair & 1 & 2 \\
\hline Poor & 2 & 4 \\
\hline
\end{tabular}

Table 6: Showing results in various Garden's grades

\begin{tabular}{|c|c|c|}
\hline Garden's grade & No. of cases & Results \\
\hline $\mathrm{I}$ & 7 & Excellent - 7(100\%) \\
\hline II & 10 & $\begin{array}{c}\text { Excellent - 8(80\%) } \\
\text { Good - } 2(20 \%)\end{array}$ \\
\hline III & 8 & $\begin{array}{c}\text { Excellent }-7(87.5 \%) \\
\text { Poor - } 1(12.5 \%)\end{array}$ \\
\hline IV & 25 & $\begin{array}{c}\text { Excellent }-16(64 \%) \\
\text { Good - } 7(28 \%) \\
\text { Fair - } 1(4 \%) \\
\text { Poor - } 1(4 \%) \\
\end{array}$ \\
\hline
\end{tabular}




\section{Conclusion}

The femoral neck fracture continues to be unsolved fracture and the guidelines for management are still evolving. It is common skeletal injury, occurring with minor trauma in the osteoporotic bone of elderly patients. In younger patients, it occurs due to high velocity trauma and may be a part of polytrauma with multiple fractures.

The management has evolved significantly. It started with close reduction and immobilisation in POP hip spica in abduction and internal rotation (Whiteman abduction plaster) in the early part of 20th century. High incidences of AVN, non-union, bed sores and respiratory complications led to exploration of methods of internal fixation. The introduction of SP nail brought a new hope of solving the problem, but high failure rate and complication rates disappointed many surgeons. Further improvement in implant designs brought in newer devices like SP nail plate and McLaughlin nail plate; these also did not stand the test of time. The modern concept of fixation under compression led to the use of partially threaded cancellous screws and placement over preliminary wires led to the development of cannulated variety of screws, which are now the standard of care in adults. Smooth pins (Moore and Knowles) are still the choice in children.

The presentation at different ages poses different problems related to the management. The issues are fixation failure in osteoporotic bone of elderly, marked displacement of fragments, posterior comminution and disruption of blood supply in adults, and a higher incidence of non-union and AVN in adults. Hence accurate reduction and internal fixation are mandatory requirements to expect fracture healing.

In the present study it was found that mean age was 36.66 years with male predominance( $78 \%$ ) and regarding site of injury left to right ratio was $1.17: 1$.

In this study, Garden's classification was used for operative evaluation. It was found that most common type of injury was type IV with 25 cases ( $50 \%$ ), followed by type II and type III. More cases of displaced fractures may be related to the force required in adults for causing neck femur fracture and most common cause of fracture being RTA.

The functional results in current study were made based on Modified Harris Hip Scoring system. The scoring system has maximum points of 100 and is analysed based on patients symptoms and functional activities at follow up. Our study showed $76 \%$ having excellent results, $18 \%$ having good results, $2 \%$ having fair and $4 \%$ poor results. It was found that there is correlation between initial displacement and the final functional scores. In our study, undisplaced fractures (grade I and II) consistently showed excellent and good results whereas displaced fractures (grade III and IV) were associated with fair and poor results also. This may be related to initial displacement at the time of injury and difficulty in achieving reduction during operative procedure.

\section{Discussion}

Many studies were carried out to find out the results of fixation in femoral neck fracture like studies by M.F. Swiontkowski et al. (1984), St. Pellet et al. (2003) ${ }^{[18], ~ G . J . ~}$ Haidukewych et al. (2004) (23), KBL et al. (2004) [19], Sibaji chaudhari (2008) ${ }^{[24]}$ and Lo Irene et al (2011) ${ }^{[20]}$, and all favours the treatment with cancellous screws for fixation of femoral neck fracture.

Preservation of femoral head with internal fixation is desirable in younger and more active patients with femoral neck fracture. A healed femoral neck fracture, without the development of osteonecrosis, leads to good functional outcome. Initial fracture displacement and disruption of femoral head blood supply are factors that are outside of surgeon's control, but the ability to achieve a good outcome by decreasing fixation failure and rate of non-union depends on several factor that surgeon can control namely, the quality of reduction and obtaining a stable fixation. In adults, femoral neck should be preserved and fixed with cancellous screws as this gives high percentage of excellent and good results. Hence this would be the best procedure for intracapsular fracture neck of femur, and this is going to stay for a long period in orthopaedic practice.

The limitations of current study were that study group is small; follow up was of medium duration to comment on true incidence of AVN. So further follow up is required to comment on true incidence of AVN.

\section{References}

1. Cooper A. A treatise of dislocation and on fractures of the joints. London, Longman, Rees, 1822.

2. Cooper A. Fractures of the upper part of the thigh bone. A treatise of dislocation and on fractures of the joints, 3rd edition London, Longman, Rees, 1824.

3. Carnesale PG, Anderson LD. Primary prosthetic replacement for femoral neck fractures. Arch. Surg. 1975; 110:27-29.

4. Whitman R. A new method of treatment for fractures of the neck of the femur, together with remarks on coxa vara. Ann Surg. 1902; 36:746-761.

5. Whitman R. The abduction method considered as the standard routine in the treatment of fractures of the neck of the femur. J Orthhop Surg. 1920; 2:547-553.

6. Leadbetter GW. Closed reduction of fractures of the neck of femur. J Bone Joint Surg. 1938; 20:108-113.

7. Wilym G, Davis G. The operative treatment of intracapsular fracture of the neck of the femur. J Bone Joint Surg Am. 1909; s2-6:481-483.

8. Smith-Peterson MN, Cave EF, Van Gorder GW. Intracapsular fractures of the neck of the femur: treatment by internal fixation. Arch Surg. 1931; 23:715-759.

9. Johansson S. The operative treatment of medial fractures of femoral neck. Acta Orthop Scand. 1932; 3:362-385.

10. Westcott HH. A method for the internal fixation of transcervical fractures of the femur. J Bone Joint Surg. $1934 ; 16: 372-378$.

11. Crawfurd E, Emery RJ, Hansell DM et al. Capsular tension and intracapsular pressure in subcapital fracture of the femur. J Bone Joint Surg. 1988; 70B:195.

12. Thaun VL, Marc Swiontkowski F. Treatment of femoral neck fractures in young adults. J Bone Joint Surg Am. 2008; 90:2254-2266.

13. Eliasson-Eiskjaer S, Ostgard SE. Survivorship analysis of hemiarthroplasty. Clin Orthop. 1993; 286:206-211.

14. Kreder HJ. Arthroplasty led to fewer failures and more complications than did internal fixation for displaced fractures of the femoral neck. J Bone Joint Surg Am. 2002; 84:2108.

15. Catto M. A histological study of avascular necrosis of the femoral head after transcervical fracture, J Bone Joint Surg Br. 1965; 47:749-776.

16. Catto M. The histological appearances of late segmental collapse of the femoral head after transcervical fracture, J Bone Joint Surg Br. 1965; 47:777-791.

17. Garden RS. Malreduction and avascular necrosis in subcapital fractures of the femur. J Bone Joint Surg Br. 1971; 53:183-196. 
18. St. Petel et al. Sub or inter-trochantric fractures following screw fixation. Swiss Surg. 2003; 9:82-86.

19. KBL Lee et al. Cancellous screw fixation for femoral neck fractures: one hundred and sixteen patients. Ann Acad Med Singapore. 2004; 33:248-251.

20. Lo Irene et al. Journal of orthopaedics trauma and rehabilitation, 2011, 1-4.

21. Terry S, James Beaty H. Campbell's operative orthopaedics: $11^{\text {th }}$ edition, 3:3277-3278.

22. Terry S, James Beaty H. Campbell's operative orthopaedics: $11^{\text {th }}$ edition, 3:3276.

23. George Haidukewych J, Walter Rothwell S, David Jacofisky J, Micheal Torchiaand E, Daniel Berry J. Operative treatment of femoral neck fractures in patients between the age of fifteen and fifty years. J Bone Joint Surg Am. 2004; 86:1711-1716.

24. Sibaji Choudhari. Closed reduction, internal fixation with quadratus femoris muscle pedicle bone grafting in displaced femoral neck fractures: Indian J Orthop. 2008; 42(1):33-38. 